\title{
Inversion Time
}

National Cancer Institute

\section{Source}

National Cancer Institute. Inversion Time. NCI Thesaurus. Code C116124.

The time between the inversion and excitation pulses in an inversion recovery pulse sequence. (CDISC) 